\title{
How Does the COVID-19 Pandemic Influence Surgical Case Load and Histological Outcome for Colorectal Cancer? A Single-Centre Experience
}

\author{
Peter Tschann ${ }^{1}$ (D) Paolo N. C. Girotti ${ }^{1}$. Daniel Lechner ${ }^{1}$. Stephanie Adler ${ }^{1}$ • Benedikt Feurstein ${ }^{1}$. \\ Philipp Szeverinski $^{2} \cdot$ Ingmar Königsrainer $^{1}$
}

Received: 22 February 2021 / Accepted: 31 March 2021 / Published online: 14 April 2021

(C) 2021 The Society for Surgery of the Alimentary Tract

Keywords COVID-19 $\cdot$ Colorectal cancer $\cdot$ Delay $\cdot$ Oncological outcome

\section{Background}

Twelve months ago, the Coronavirus disease 2019 (COVID-19) broke out. Delays in cancer diagnosis or therapy are a serious concern and may result in decreased survival for solid malignancies ${ }^{1}$. Previous literature is showing conflicting results regarding the survival of delayed therapy of colorectal cancer (CRC) ${ }^{1-3}$. Tumour progression caused by a delay in diagnostics has potentially dramatic consequences for patients. The aim of this study was to investigate how a delay in diagnostics due to the pandemic influences surgical CRC case load and histological outcome compared to the time before the pandemic started.

\section{Methods}

All surgical CRC cases that underwent resection beginning on 1 January 2019 and ending on 31 December 2020 were retrieved from the electronic database at our hospital. Patients' characteristics, surgical technique, tumour location and urgency of surgery were recorded. The pathological examination included a complete histopathological staging according to TNM stage. All patients received a CT scan of the trunk before (elective cases) or

Peter Tschann and Paolo N. C. Girotti contributed equally to this work.

Peter Tschann

peter.tschann@lkhf.at

1 Department of General and Thoracic Surgery, Academic Teaching Hospital Feldkirch, Carinagasse 47, A-6800 Feldkirch, Austria

2 Institute of Medical Physics, Academic Teaching Hospital Feldkirch, Feldkirch, Austria after surgery (emergency procedures) to determinate the UICC stage. Statistical analysis was performed using the SPSS (IBM, New York). Continuous data are represented as average $( \pm \mathrm{SD})$. Significance was set at a $p$ value of $<0.05$.

\section{Results}

Results are shown in Table 1 and Fig. 1. In total, 134 patients (76 male and 58 female) underwent surgery because of colorectal cancer. Comparing the halves of the year in 2019 and 2020, we observed no difference regarding patients' characteristics. We performed significantly fewer procedures during the first lockdown compared to the same period in $2019(2019, n=$ 14 vs. $2020, n=4 ; p=0.02)$. Overall, the number of surgical CRC cases a year were comparable in $2019(n=71)$ and 2020 $(n=63)$. In $2.9 \%(n=4)$, we observed a pTis, in $6.7 \%(n=9)$ a pT1, in $14.9 \%(n=20)$ a pT2, a pT3 in $41.0 \%(n=55)$ and a pT4 tumour in $34.3 \%(n=46)$. We observed a UICC stage 0 in $2.2 \%(n=3)$, a UICC stage I in $18.6 \%(n=25)$, a UICC stage II in $26.8 \%(n=36)$, a UICC stage III in $29.8 \%(n=40)$ and a UICC stage IV in $22.3 \%(n=30)$. If a neoadjuvant treatment was performed, the preoperatively UICC stage was considered. We observed - not significantly - more T4 stages in the first and second half of 2020 compared to the same time one year before $(p=0.47)$. Complication rates did not differ during 2019 and $2020(p=0.58)$.

\section{Discussion}

Our study clearly showed a significant decrease of surgical CRC cases during the first lockdown from 16 March to 26 
Table 1 Patient's characteristics (A), perioperative results (B) and oncological results (C)

\begin{tabular}{|c|c|c|c|c|c|c|c|c|c|}
\hline \multicolumn{10}{|l|}{ A: Patients' characteristics } \\
\hline & \multicolumn{2}{|c|}{ 1st half of the year 2019} & \multicolumn{3}{|c|}{ 2nd half of the year 2019} & \multicolumn{2}{|c|}{ 1st half of the year 2020} & \multicolumn{2}{|c|}{ 2nd half of the year 2020} \\
\hline$n(=134)$ & \multicolumn{2}{|c|}{46} & \multicolumn{3}{|c|}{25} & \multicolumn{2}{|c|}{29} & 34 & 0.06 \\
\hline $\operatorname{Sex}(m / w)$ & $27 / 19$ & & $15 / 10$ & & & $12 / 17$ & & $22 / 12$ & 0.28 \\
\hline Age (years) & $67.5( \pm 11.7)$ & & $64.0( \pm 15.7)$ & & & $69.5( \pm 13.1)$ & & $68.1( \pm 13.2)$ & 0.63 \\
\hline *BMI $\left(\mathrm{kg} / \mathrm{m}^{2}\right)$ & $25.2( \pm 4.7)$ & & $25.2( \pm 4.8)$ & & & $24.3( \pm 3.5)$ & & $27.3( \pm 5.3)$ & 0.11 \\
\hline$* *$ ASA [], $n(\%)$ & & & & & & & & & 0.95 \\
\hline I & 3 & $6.5 \%$ & 4 & $16.0 \%$ & 4 & $13.8 \%$ & 6 & $17.6 \%$ & 0.39 \\
\hline II & 23 & $50.0 \%$ & 12 & $48.0 \%$ & 14 & $48.3 \%$ & 14 & $41.2 \%$ & 0.89 \\
\hline III & 17 & $37.0 \%$ & 8 & $32.0 \%$ & 10 & $34.5 \%$ & 12 & $35.3 \%$ & 0.99 \\
\hline IV & 3 & $6.5 \%$ & 1 & $4.0 \%$ & 1 & $3.4 \%$ & 2 & $5.9 \%$ & 1.00 \\
\hline \multicolumn{10}{|l|}{ B: Perioperative results } \\
\hline Duration of hospital stay (days) & \multicolumn{2}{|l|}{$13.6( \pm 9.1)$} & \multicolumn{2}{|l|}{$14.9( \pm 17.2)$} & \multicolumn{2}{|c|}{$12.2( \pm 7.8)$} & \multicolumn{2}{|c|}{$15.0( \pm 13.5)$} & 0.91 \\
\hline Emergency cases, $n(\%)$ & 2 & $4.3 \%$ & 7 & $28.0 \%$ & 5 & $17.2 \%$ & 7 & $20.6 \%$ & 0.03 \\
\hline Laparoscopy, $n(\%)$ & 24 & $52.2 \%$ & 12 & $48.0 \%$ & 11 & $37.9 \%$ & 17 & $50.0 \%$ & 0.59 \\
\hline Open, $n(\%)$ & 22 & $47.8 \%$ & 13 & $52.0 \%$ & 18 & $62.1 \%$ & 17 & $50.0 \%$ & 0.40 \\
\hline \multicolumn{10}{|l|}{ Localisation, $n(\%)$} \\
\hline Right colon + flexure & 16 & $34.8 \%$ & 9 & $36.0 \%$ & 9 & $31.0 \%$ & 16 & $47.1 \%$ & 0.58 \\
\hline Transverse colon & 1 & $2.2 \%$ & 2 & $8.0 \%$ & 5 & $17.2 \%$ & 1 & $2.9 \%$ & 0.07 \\
\hline Left colon + flexure & 5 & $10.9 \%$ & 2 & $8.0 \%$ & 1 & $3.4 \%$ & 1 & $2.9 \%$ & 0.52 \\
\hline Sigmoid colon & 7 & $15.2 \%$ & 8 & $32.0 \%$ & 8 & $27.6 \%$ & 7 & $20.6 \%$ & 0.34 \\
\hline Rectum & 17 & $37.0 \%$ & 4 & $16.0 \%$ & 6 & $20.7 \%$ & 9 & $26.5 \%$ & 0.24 \\
\hline Complications: & 9 & $19.6 \%$ & 5 & $20.0 \%$ & 3 & $10.3 \%$ & 8 & $23.5 \%$ & 0.58 \\
\hline Leakage & 6 & $13.0 \%$ & 4 & $16.0 \%$ & & $0.0 \%$ & 3 & $8.8 \%$ & 0.13 \\
\hline Fascial dehiscence & 2 & $4.3 \%$ & & $0.0 \%$ & 2 & $6.9 \%$ & 2 & $5.9 \%$ & 0.73 \\
\hline Bleeding & & $0.0 \%$ & & $0.0 \%$ & & $0.0 \%$ & 2 & $5.9 \%$ & 0.14 \\
\hline Stoma dehiscence & & $0.0 \%$ & 1 & $4.0 \%$ & & $0.0 \%$ & & $0.0 \%$ & 0.19 \\
\hline Aspiration & & $0.0 \%$ & & $0.0 \%$ & & $0.0 \%$ & 1 & $2.9 \%$ & 0.66 \\
\hline Peritonitis & 1 & $2.2 \%$ & & $0.0 \%$ & & $0.0 \%$ & & $0.0 \%$ & 1.00 \\
\hline Influenca pneumonia & & $0.0 \%$ & & $0.0 \%$ & 1 & $3.4 \%$ & & $0.0 \%$ & 0.40 \\
\hline COVID-19 pneumonia & & $0.0 \%$ & & $0.0 \%$ & & $0.0 \%$ & 1 & $2.9 \%$ & 0.66 \\
\hline \multicolumn{10}{|c|}{ C: Oncological results (*in case of neoadjuvant therapy, preoperatively measured UICC stage was considered) } \\
\hline$* \mathrm{UICC}[], n(\%)$ & & & & & & & & & 0.79 \\
\hline 0 & 1 & $2.2 \%$ & 1 & $4.0 \%$ & 0 & $0.0 \%$ & 1 & $2.9 \%$ & 0.88 \\
\hline 1 & 10 & $21.7 \%$ & 3 & $12.0 \%$ & 4 & $13.8 \%$ & 8 & $23.5 \%$ & 0.61 \\
\hline 2 & 16 & $34.8 \%$ & 6 & $24.0 \%$ & 8 & $27.6 \%$ & 6 & $17.6 \%$ & 0.43 \\
\hline 3 & 12 & $26.1 \%$ & 7 & $28.0 \%$ & 10 & $34.5 \%$ & 11 & $32.4 \%$ & 0.85 \\
\hline 4 & 7 & $15.2 \%$ & 8 & $32.0 \%$ & 7 & $24.1 \%$ & 8 & $23.5 \%$ & 0.42 \\
\hline Т [], $n(\%)$ & & & & & & & & & 0.65 \\
\hline 0 & 2 & $4.3 \%$ & 1 & $4.0 \%$ & 0 & $0.0 \%$ & 1 & $2.9 \%$ & 0.83 \\
\hline 1 & 3 & $6.5 \%$ & 2 & $8.0 \%$ & 0 & $0.0 \%$ & 4 & $11.8 \%$ & 0.31 \\
\hline 2 & 8 & $17.4 \%$ & 2 & $8.0 \%$ & 5 & $17.2 \%$ & 5 & $14.7 \%$ & 0.76 \\
\hline 3 & 20 & $43.5 \%$ & 13 & $52.0 \%$ & 12 & $41.4 \%$ & 10 & $29.4 \%$ & 0.36 \\
\hline 4 & 13 & $28.3 \%$ & 7 & $28.0 \%$ & 12 & $41.4 \%$ & 14 & $41.2 \%$ & 0.47 \\
\hline $\mathrm{N}[], n(\%)$ & & & & & & & & & 0.77 \\
\hline 0 & 30 & $65.2 \%$ & 12 & $48.0 \%$ & 18 & $62.1 \%$ & 20 & $58.8 \%$ & 0.56 \\
\hline 1 & 8 & $17.4 \%$ & 4 & $16.0 \%$ & 5 & $17.2 \%$ & 6 & $17.6 \%$ & 1.00 \\
\hline 2 & 8 & $17.4 \%$ & 9 & $36.0 \%$ & 6 & $20.7 \%$ & 8 & $23.5 \%$ & 0.36 \\
\hline Resected lymph nodes, $n$ & $17.63( \pm 5.18)$ & $18.56( \pm 9.01)$ & $17.55( \pm 6.17)$ & $15.89( \pm 6.87)$ & 0.72 & & & & \\
\hline
\end{tabular}

m, men; w, women; UICC, Union Internationale Contre le Cancer; BMI, body mass index; ASA, American Society of Anaesthesiologists Classification

April 2020, compared to the same period in 2019. This may be caused by the complete absence of routine diagnostic during the first lockdown and because of a reduction of operation room resources ${ }^{4}$. The second lockdown was more selective and routine diagnostic tools were available. Comparing the periods in 2019 and 2020, we did not observe a difference regarding patients' characteristics, type of procedure and urgency of surgery compared to the time before the pandemic started. Oncological outcomes were comparable in the analysed periods. A change of the management in rectal cancer therapy was not necessary because of COVID19Klicken oder tippen Sie hier, um Text einzugeben.. Interestingly, we observed a non-significant increase of T4 stages in the first and second half of 2020 compared to the same time in 2019. This study is limited by its retrospective character and single institution experience. To rule out statistical difficulties due to the relatively small number of cases in this study, a multicentre trial and register data would be helpful. 
a

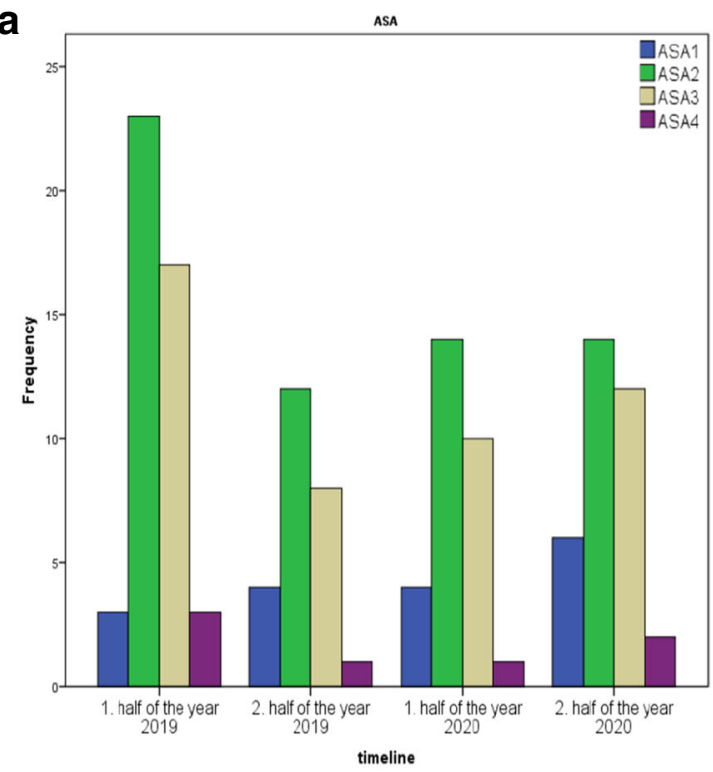

C

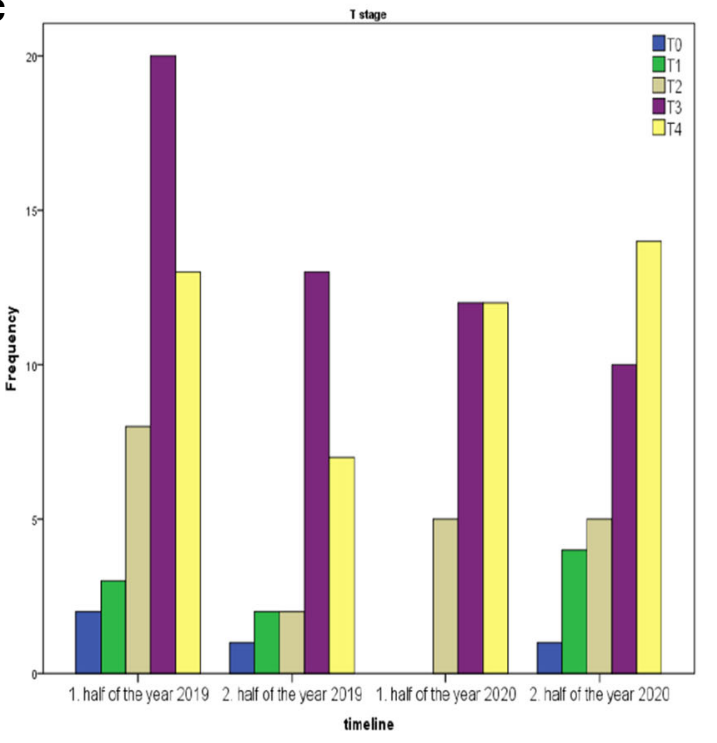

Fig. 1 A ASA Classification (American Society of Anaesthesiology Classification): Horizontal axis shows the timeline, the ordinate is showing the frequency of procedures. B Timeline and number of procedures per month in 2020. The two lockdowns are highlighted. Number of cases decreased during the first lockdown. C Local tumor

Acknowledgement The authors like to thank Simone Minikus for English and spelling corrections.

Author Contributions P.T., P.G., D.L., S.A., B.F., P.S. and I.K. designed the study. Collection of data was done by P.T. P.T. wrote the manuscript, P.T. and P.S. prepared the tables. P.S. did the statistical analysis. All authors contributed towards data acquisition, data interpretation and critical revision of the content of the manuscript and approved the final version of the manuscript.

Data Availability The datasets generated and/or analysed during the current study are available from the corresponding author on reasonable request.
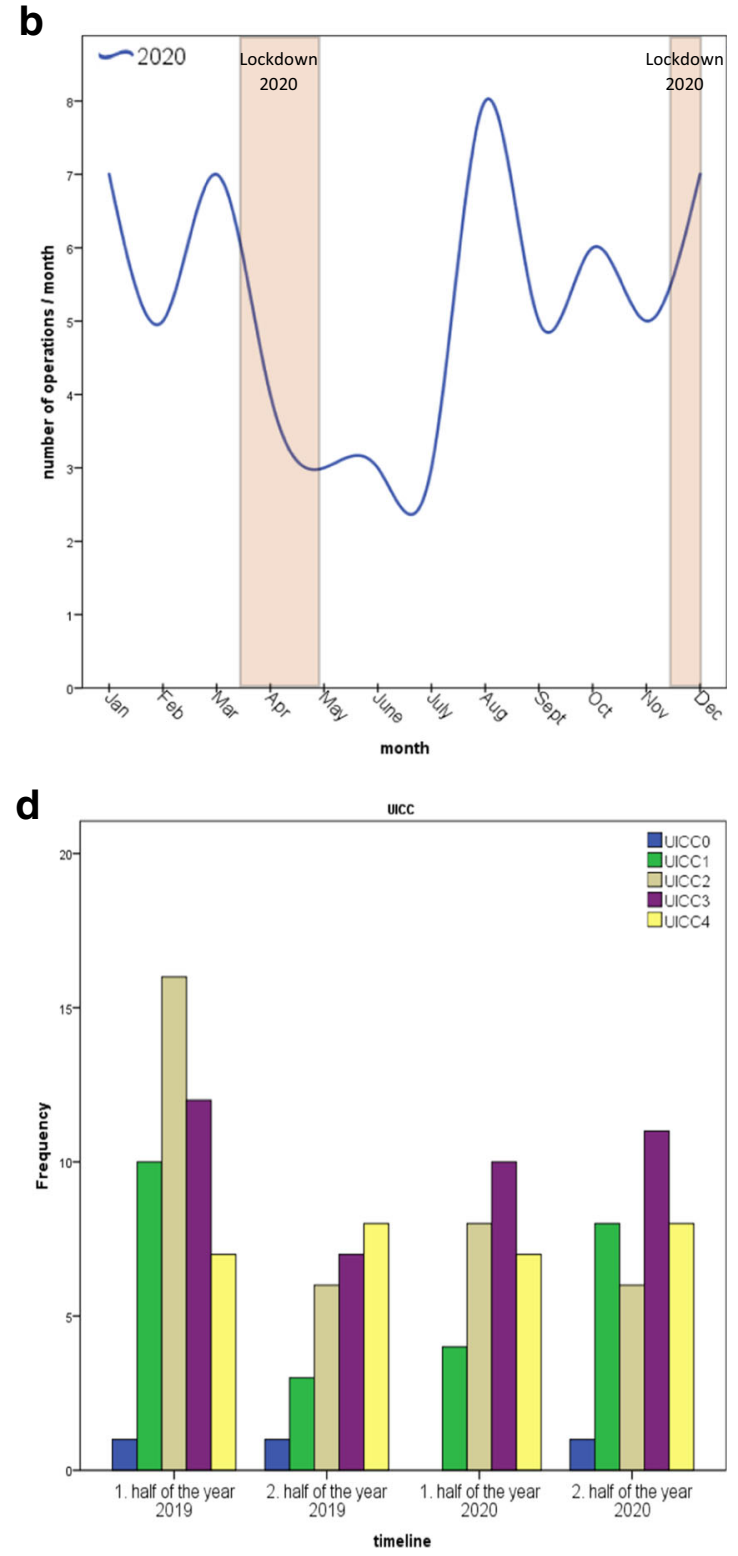

stage (pT): Horizontal axis shows the timeline, the ordinate is showing the frequency of procedures. D UICC-Classification (Union internationale contre le cancer): Horizontal axis shows the timeline, the ordinate is showing the frequency of procedures

\section{Declarations}

Ethics Approval This study was presented to the Ethics Committee of the Province of Vorarlberg and does not require any referral or vote by this committee (EK-0.04-340).

\section{Consent of Participate n/a}

Consent of Publication n/a

Conflict of Interest P.T., P.N.C.G., D.L., S.A., B.F., P.S., and I.K. have no conflict of interest or financial ties to disclose. 


\section{References}

1. Grass F, Behm KT, Duchalais E, Crippa J, Spears GM, Harmsen WS et al. Impact of delay to surgery on survival in stage I-III colon cancer. European journal of surgical oncology the journal of the European Society of Surgical Oncology and the British Association of Surgical Oncology 2020;46(3):455-61.

2. Sud A, Jones ME, Broggio J, Loveday C, Torr B, Garrett A et al. Collateral damage: the impact on outcomes from cancer surgery of the COVID-19 pandemic. Annals of oncology official journal of the European Society for Medical Oncology 2020;31(8):1065-74.
3. Trepanier M, Paradis T, Kouyoumdjian A, Dumitra T, Charlebois P, Stein BS et al. The impact of delays to definitive surgical care on survival in colorectal cancer patients. Journal of gastrointestinal surgery official journal of the Society for Surgery of the Alimentary Tract 2020;24(1):115-22.

4. Lechner D, Tschann P, Girotti PCN, Königsrainer I. Impact of the COVID-19 pandemic on a visceral surgical department in western Austria. European surgery ACA Acta chirurgica Austriaca 2020:15 .

Publisher's Note Springer Nature remains neutral with regard to jurisdictional claims in published maps and institutional affiliations. 